\title{
A simplified sphere decoding algorithm for MIMO transmission system
}

\begin{abstract}
In sphere decoding the choice of sphere radius is crucial to excellent performance. In ChanLee sphere decoding -based algorithm, the problem of choosing initial radius has been solved by making the radius sufficiently large, thus increasing the size of the search region. In this paper we present maximum likelihood decoding using simplified sphere decoder as apposed to the original sphere decoder for the detection of cubic structure quadrature amplitude modulation symbols. This simple algorithm based on Chan-Lee sphere decoder allows the search for closest lattice point in a reduced complexity manner compared to original sphere decoder for multiple input multiple output system with perfect channel state information at the receiver. Results show symbol error rate has stabilized even at very low initial value of the square radius.
\end{abstract}

Keyword: Simplified sphere decoder; Chan-Lee algorithm; MIMO transmission system 